\title{
Analysis of C-shaped Compact Microstrip Antennas Using Deep Neural Networks Optimized by Manta Ray Foraging Optimization with Lévy-Flight Mechanism
}

\author{
Mustafa Berkan Biçer ${ }^{1}$ \\ ${ }^{1}$ Department of Electrical and Electronics Engineering, Izmir Bakircay University, Izmir, Turkey; \\ mustafa.bicer@bakircay.edu.tr; +90 (232) 4930000 / 11237
}

Received 25 March 2021; Revised 27 April 2021; Accepted 29 April 2021; Published online 31 August 2021

\begin{abstract}
In recent years, microstrip antennas have become a popular research subject with the increasing use of mobile technologies. With the development of neural networks, the design and analysis of microstrip antennas are carried out quickly with high accuracy. However, optimizing the weight matrices and bias vectors of deep neural learning models is an important challenge for engineering problems. This study presents a deep neural network-based (DNN-based) neural model to estimate the gain and scattering parameter $\left(\mathrm{S}_{11}\right)$ of C-shaped compact microstrip antennas (CCMAs). For this purpose, the $S_{11}$ and gain values of 324 CCMAs with different physical and electrical properties were obtained using full-wave electromagnetic simulation software based on the finite integration technique (FIT). The data related to 324 CCMAs were used for the training and testing process. The improved manta ray foraging optimization (MRFO) algorithm based on the Lévy-flight (LF) mechanism was used to optimize the connection weights matrices and bias vectors. The MRFO-optimized model has estimation success for training and testing data as 0.925 and 0.922 , in terms of $\mathrm{R}^{2}$ score, respectively. The estimated resonant frequencies using the trained model are compared with the studies in the literature, and an average percentage error (APE) of $0.933 \%$ is obtained.
\end{abstract}

Keywords: C-shaped microstrip antenna, deep neural networks, manta ray foraging optimization, lévy flight technique, S-parameter estimation, gain estimation

\section{Introduction}

With the rapid development of technology, small-sized wireless and portable devices such as navigation devices, headphones, cameras, and mobile phones and have found a wider area of use in daily life. Microstrip antennas (MA) [1, 2] are essential components of the systems such as wireless communication, spacecraft, aircraft, radars, satellite communication, and guided missiles. MAs have superior advantages, including ease in production, small in size, integrated use with electronic circuit boards, and solid-state devices [2-6]. Recent advances in mobile communication technology have increased the need for MAs operating at lower frequency bands. Due to its nature, the reduction in the physical dimensions of the antenna causes an increase in the resonant frequency of the antenna. However, the resonant frequency of conventional-shaped MAs such as circular, triangular, and rectangular can be reduced by modifying the structures [2, 5]. Compact MAs (CMAs) are formed by modifying the geometries of conventional-shaped MAs [2, 7]. Although theoretical methods, including the transmission-line model (TLM) [8] and cavity model [9], are used to analyze conventional-shaped MAs, these methods have various difficulties for CMAs due to their complex structure and non-linear behavior. Therefore, the analysis of CMAs is generally carried out using full-wave electromagnetic solvers based on numerical methods such as finite-difference time-domain (FDTD) [10, 11], finite element method (FEM) [12], finite integration technique (FIT) [13, 14], and method of moments (MoM) $[15,16]$. In recent years, researchers have vigorously challenged the accurate calculation of the resonant frequency of CMAs. While most studies focus on designing and optimizing CMA structures [3, 17-20] and obtaining mathematical models for determining the resonant frequencies of the antennas [5, 7, 2124], there are also studies on computing the resonant frequencies or other characteristic parameters using neural network models [25-34]. In most recent studies, resonant frequency estimation was made in two ways: the closed-form expression approach and the neural model approach. Akdagli et al. [7] have 
proposed a closed-form resonant length expression to compute the dominant operating frequency of Cshaped compact microstrip antennas (CCMAs). To optimize the mathematical model, the authors have designed and simulated 144 CCMAs with various physical and electrical properties and utilized the artificial bee colony (ABC) algorithm [35-37]. In [23], mathematical expressions for resonant lengths of C-shaped and H-shaped CMAs are presented. Toktas et al. made simulations for certain parameter ranges, and the unknown variables of the expressions were determined employing the ABC algorithm [35-37]. Biswas and Dam [24] have introduced a closed-form mathematical model to calculate the probe reactance. In their study [24], an equilateral triangular patch antenna was fabricated, and the calculated resonant frequencies were compared with the measured and simulated resonant frequencies. A further equilateral triangle-shaped compact microstrip antenna study has been carried out by Kapusuz et al. [31]. In [31], the authors presented an artificial neural network (ANN) to determine the resonant frequency of equilateral triangular patch antennas for different $\mathrm{TM}_{\mathrm{mnp}}$ modes. Can et al. have proposed a multilayer perceptron (MLP) neural network (NN) model to calculate the operating frequency of coaxial-fed pin-loaded rectangular microstrip patch antennas [32]. The proposed MLPNN model [32] uses the physical and electrical properties of the antenna as input and estimates the upper and lower operating frequency of the antenna. Abbassi et al. [38] have designed a CMA operating at a frequency of 2.4 GHz and used a NN model to predict the $\mathrm{S}_{11}$ and gain parameters. Neebha and Nesasudha [34] have designed a CMA for C-band applications using an ANN model. Four antenna-related parameters were used as the network input, and two physical parameters were estimated [34]. Singh et al. [33] studied on a coaxial-fed E-shaped CMA (ECMA) operating at the $2.4 \mathrm{GHz}$ frequency. In [33], to analyze the performances of the neural network models, the $\mathrm{x}$ - and $\mathrm{y}$ - coordinates of the coaxial feed were used as input, and resonant frequency, $\mathrm{S}_{11}$ value, VSWR value, and input impedance parameters were chosen as output. Sami et al. have proposed an ANN model to estimate the resonant frequency and quality factor of a circular microstrip antenna in the $\mathrm{TM}_{11}$ mode [25]. The authors have used isotropic substrate and uniaxially anisotropic substrate in MA design [25]. In another study, a neural model for the synthesis and analysis of rectangular MAs using uniaxially anisotropic substrates was developed by Barkat et al. [26]. The patch dimensions were used for synthesis, and the resonant frequency and bandwidth parameters were used for analysis [26]. In [30], Kaur and Sivia have proposed a miniaturized hybrid fractal antenna based on Giuseppe Peano and Cantor set fractals for biomedical systems. While the firefly algorithm (FA) [36, 39] was used to optimize the feeding point of the antenna [30], ANN was employed in the calculation of the two resonant frequencies. In a recently published study [29], a deep neural network-based (DNN-based) model consisting of five layers, three of which are hidden layers, has been proposed by Ustun et al. to calculate the resonant frequency of ECMAs. The authors simulated 144 ECMAs and trained the neural model using the simulated data [29]. As mentioned above, several studies on the calculation or estimation of the resonance frequency of MAs have been done in the literature, but there is still insufficient study for the $S_{11}$ and gain estimation. Studies on neural networks in the literature generally focused on estimating the resonance frequencies of antennas and their gains at this frequency. However, in this study, the estimation of $S_{11}$ and gain values of the antenna not at a single frequency but in a certain frequency range was studied. $S_{11}$ is a critical parameter that indicates whether the antenna carries the desired radiation performance in a frequency range and whether impedance matching is achieved in this range. The gain is also an important parameter as it takes the losses into account, representing the transmitted power in any direction with regard to an isotropic antenna. Hence, this study is focused on obtaining the $S_{11}$ and gain curves of CCMAs designed. It is seen from the literature that the computer-aided design (CAD) tools and machine learning-based models are of great importance in guiding the antenna design process.

In this study, a DNN-based neural model is proposed to estimate the $S_{11}$ and gain parameter of CCMAs for a frequency range. CCMAs with a specific value range of physical and electrical parameters were designed and simulated using a full-wave electromagnetic solver based on FIT. The obtained $\mathrm{S}_{11}$ and the gain values for the desired frequency range were used to build a data set. The neural model was trained by utilizing the improved manta ray foraging optimization (MRFO) algorithm [40] based on the Lévy-flight (LF) mechanism [36, 41]. The estimated values were compared with the studies in the literature. 
This paper is divided into six sections. In the first section, the definition of the problem is given by doing a literature review. The covers the antenna design and simulation are covered in the second section, and the third section introduces the manta ray foraging optimization algorithm and Lévy-flight mechanism. The neural model structure and the training process are included in the fourth section, while the numerical results and comparisons are given in the fifth section. In the last section, discussions about the study are mentioned.

\section{Design and Simulation of the C-shaped Compact Microstrip Antennas}

A C-shaped compact microstrip antenna (CCMA) consists of a dielectric substrate and two thin copper planes placed on either side of this substrate, as shown in Figure 1. The C-shape was formed by removing a portion having the dimensions of $l \times w$ from the right side of the radiating copper to make the shape of the plane similar to the letter- $C$. While the radiating $C$-shaped copper plane was placed on top of the substrate having a dielectric constant $\varepsilon_{r}$ and thickness $h$, the ground plane covered the bottom. Coaxial feeding was chosen as the feeding technique for the convenience of performing parametric simulation studies. A $50-\Omega$ probe was used to feed the CCMA at the point of $(0.1 L, 0.1 W)$ for all CCMA designs.

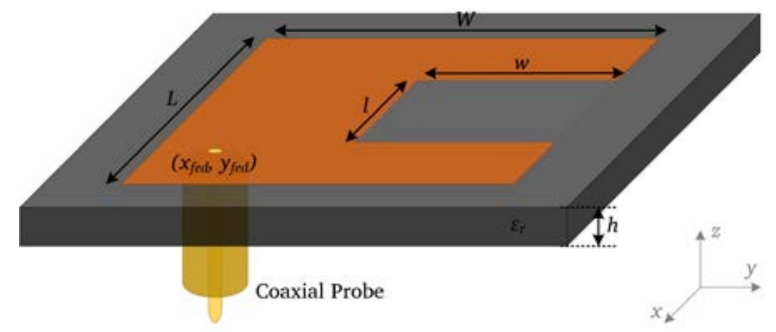

Figure 1 Three-dimensional representation of the coaxial-fed CCMA

In MA designs, the $h$ parameter is generally chosen to be much smaller than the free-space wavelength $\left(\lambda_{0}\right)$, as shown in Figure 1. In this case, the fringing field along the patch edges is minimal, while the electric field is almost normal along the patch surface [2]. Thus, as long as $h<<\lambda_{0}$, transverse magnetic (TM) field configurations are considered for MAs, whereas with increasing operating frequency, different field configurations may arise [2]. For $\mathrm{TM}_{\text {mnp }}$ mode, the resonant frequency of a conventional rectangular MA (RMA) is calculated by Equation 1 [2].

$$
\left(f_{r}\right)_{m n p}=\frac{v_{0}}{2 \pi \sqrt{\mu_{r} \varepsilon_{r}}} \sqrt{\left(\frac{m \pi}{h}\right)^{2}+\left(\frac{n \pi}{L}\right)^{2}+\left(\frac{p \pi}{W}\right)^{2}}
$$

In Equation 1, the indices of the radiation mode, dielectric constant, magnetic permeability, and the velocity of the electromagnetic wave in free space are represented by $(m, n, p), \varepsilon_{r}, \mu_{r}$, and $v_{0}$, respectively. The $\mu_{r}$ variable defines the ferromagnetism level and can be taken as one for substrates used for the production of the MAs. Thus, in determining the resonant frequency of RMAs, the parameters $L, W, h$, and $\varepsilon_{r}$ play essential roles. Since the mode of the lowest order resonant frequency is defined as the dominant mode, the mode orders are determined by placing the resonant frequencies in ascending order. When the condition $L>W>h$ is satisfied, the lowest order resonance frequency for dominant mode $\mathrm{TM}_{010}$ is obtained as given in Equation 2 [2].

$$
\left(f_{r}\right)_{010}=\frac{v_{0}}{2 L \sqrt{\varepsilon_{r}}}
$$

Considering Equation 2, the boundaries of the physical parameters of CCMAs were chosen so that the resonance frequency remained within the simulated frequency band range in the dominant mode. For CCMAs, $l$ and $w$ parameters were critical as they had caused electrical extension along the $L$ - and $W$ - 
axes. The outer dimensions of $L \times W$, the slot dimensions of $l \times w$, the substrate thickness of $h$, and the dielectric constant of the substrate $\varepsilon_{r}$ were considered for CCMAs. The $L$ and $l$ represented the lengths, while the widths were represented by $W$ and $w$ parameters. For the sake of simplicity, the impact of the feeding point on the $S_{11}$ and gain parameters was neglected. The physical and electrical parameters of the CCMA, $S_{11}$, and gain values for a specific frequency range were compulsory for training and testing the neural model. It was also important to choose a sufficient solution space for the proposed model to be trained. In order to achieve better results, the values should be chosen from a wide range of solutions, but simulating the entire solution area may take a long time. In this study, the values were chosen in a sufficiently wide range to ensure simplicity in simulation and training time. In order to sufficiently represent the solution space for the proposed model, the values of the thickness $(h)$ and dielectric constant $\left(\varepsilon_{r}\right)$ of the substrate, and the outer and inner dimensions of the radiating plane were chosen as shown in Table 1.

Table 1 Physical and electrical parameters and values of designed CCMAs

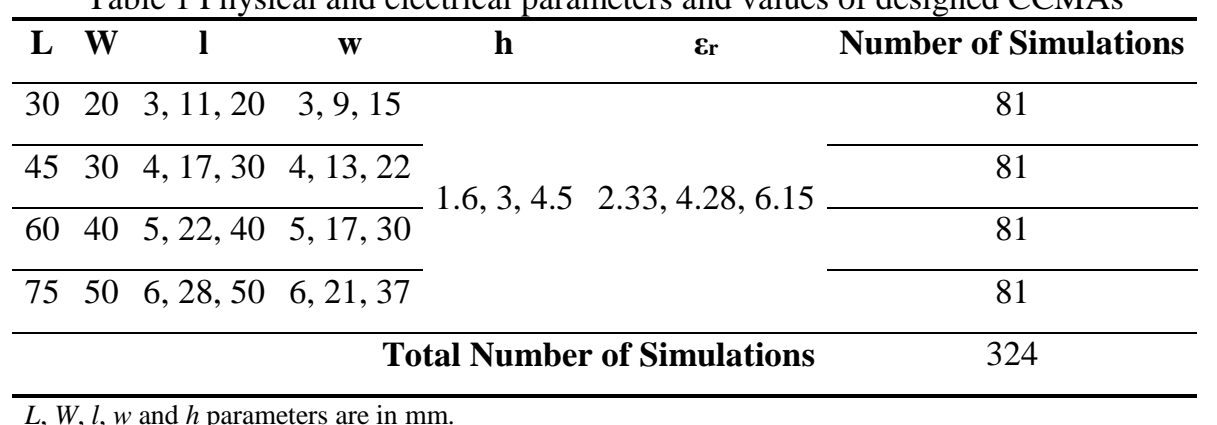

The values of $l, w, h$, and $\varepsilon_{r}$ parameters shown in Table 1 were chosen to have 81 CCMA designs for each $L \times W$ pair. The height $(h)$ and dielectric constant $\left(\varepsilon_{r}\right)$ values were selected from the standard values provided by the substrate manufacturers. Depending on the technique-specific parameters of the FITbased solver, each simulation session took approximately 5.1 minutes on a computer having an Intel Core i7-9750H processor and 32 GB RAM. After the simulation process, the gain and $S_{11}$ values were extracted from the results to generate a data set. Afterward, a seven-layered DNN-based neural model was modeled by seven inputs and two outputs. Statistical calculations, including as coefficient of determination $\left(\mathrm{R}^{2}\right)$ score, mean absolute error (MAE) and mean squared error (MSE), were carried out using the gain and $S_{11}$ values to measure training and testing performances. The followed topology is shown in Figure 2.

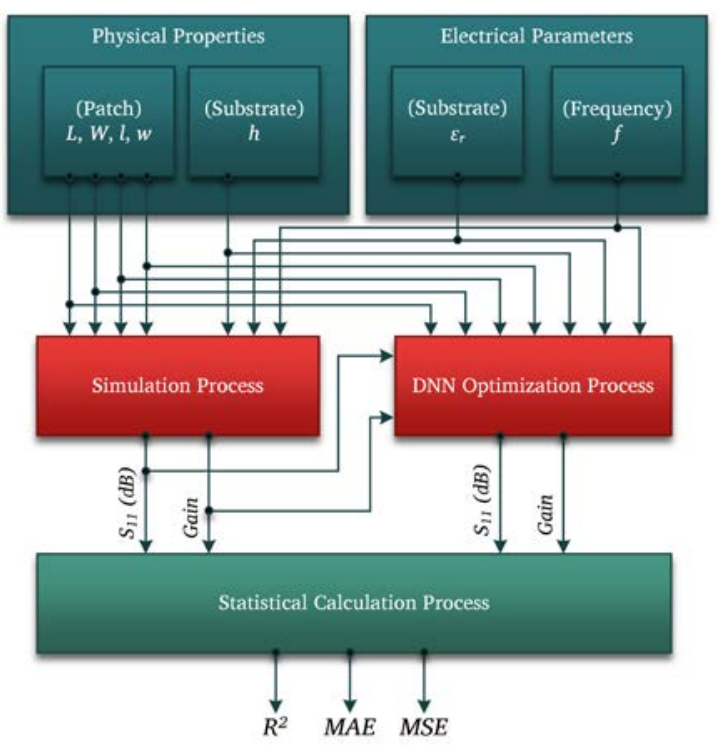

Figure 2 The topology of the processes used in the study 


\section{Overview of the Manta Ray Foraging Optimization (MRFO) Algorithm}

The intelligent behavior of living beings has become an exciting research subject for researchers in recent years [35-37, 39, 40, 42]. One of the most recently introduced bio-inspired metaheuristic optimization techniques is the Manta Ray Foraging Optimization (MRFO) algorithm [40], which searches near-optimum solutions to complex engineering problems by imitating the intelligent foraging behaviors of manta rays. Manta rays are creatures that feed on plankton in the ocean and are highly skilled in finding abundant plankton resources. Manta rays can travel on their own as well as with groups of up to 50 members, but foraging is usually done in groups. Manta rays use three different mechanisms during the foraging phase, including chain foraging, cyclone foraging, and somersault foraging.

\subsection{Chain Foraging}

Chain foraging strategy is one of the foraging strategies of manta rays to explore resources that contain abundant plankton [40]. In this group based foraging strategy, manta rays are lined up head-to-tail towards the high dense plankton source. Thanks to chain sequencing, manta rays obtain the highest efficiency from the current position by allowing plankton missed by previous individuals to be caught by the next individuals in the population. The foremost manta ray travels freely to the high-density source of plankton, while other individuals follow those in front of them. The concentration level of plankton determines the position quality. In the MRFO algorithm [40], the positions of the individuals are updated by using Equation 3 according to the chain foraging strategy. Equation 3a is used to revise the position of the first individual in the population, while Equation $3 \mathrm{~b}$ is used to update the remaining positions [40].

$$
x_{i}^{d}(t+1)=\left\{\begin{array}{cl}
x_{i}^{d}(t)+r \cdot\left(x_{\text {best }}^{d}(t)-x_{i}^{d}(t)\right)+\alpha \cdot\left(x_{\text {best }}^{d}(t)-x_{i}^{d}(t)\right) & i=1 \\
x_{i}^{d}(t)+r \cdot\left(x_{i-1}^{d}(t)-x_{i}^{d}(t)\right)+\alpha \cdot\left(x_{\text {best }}^{d}(t)-x_{i}^{d}(t)\right) & i=2, \ldots, N \\
\alpha=2 \cdot r \cdot \sqrt{|\log (r)|} &
\end{array}\right.
$$

In Equation 3a and 3b, the variables $x, r$, and $\alpha$ and the indices $d, t, i$, and best represent the position, a random value between $(0,1)$, weight coefficient, dimension, time, individual, and the highest concentrated position, respectively. The weight coefficient $\alpha$ used in Equation 3 is calculated using Equation 4 [40].

\subsection{Cyclone Foraging}

When manta ray groups discover plankton-dense positions in the deep waters, they travel to these positions with a unique strategy, called the cyclone strategy [40]. In this strategy, the manta rays are lined up as in the chain foraging strategy, and then each individual follows a spiral route while following the previous individuals. During the cyclone foraging mechanism, the positions of the individuals in the population are updated using Equation $5 \mathrm{a}$ for the first individual and Equation $5 \mathrm{~b}$ for the other individuals [40].

$$
\begin{aligned}
& x_{i}^{d}(t+1)= \begin{cases}x_{\text {best }}^{d}(t)+r \cdot\left(x_{\text {best }}^{d}(t)-x_{i}^{d}(t)\right)+\beta \cdot\left(x_{\text {best }}^{d}(t)-x_{i}^{d}(t)\right) & i=1 \\
x_{\text {best }}^{d}(t)+r \cdot\left(x_{i-1}^{d}(t)-x_{i}^{d}(t)\right)+\beta \cdot\left(x_{\text {best }}^{d}(t)-x_{i}^{d}(t)\right) & i=2, \ldots, N\end{cases} \\
& \beta=2 \cdot e^{r_{1} \frac{T-t+1}{T}} \cdot \sin \left(2 \pi r_{1}\right)
\end{aligned}
$$

The variable $\beta$ in Equation 5, and the variables $T$ and $r_{1}$ in Equation 6 represent the weight coefficient, the maximum number of iterations, and a random number between 0 and 1 , respectively. The exponential and sinusoidal terms in Equation 6 form the spiral route that is the basis of the cyclone strategy. By using Equation 7 and Equation 8, this foraging mechanism can be extended to allow each individual to randomly search for a better location by reference to their own best position to improve the global search performance of the MRFO algorithm [40]. 


$$
x_{i}^{d}(t+1)=\left\{\begin{array}{cl}
x_{\text {rand }}^{d}=L b^{d}+r \cdot\left(U b^{d}-L b^{d}\right) & \\
x_{\text {rand }}^{d}+r \cdot\left(x_{\text {rand }}^{d}-x_{i}^{d}(t)\right)+\beta \cdot\left(x_{\text {rand }}^{d}-x_{i}^{d}(t)\right) & i=1 \\
x_{\text {rand }}^{d}+r \cdot\left(x_{i-1}^{d}(t)-x_{i}^{d}(t)\right)+\beta \cdot\left(x_{\text {rand }}^{d}-x_{i}^{d}(t)\right) & i=2, \ldots, N
\end{array}\right.
$$

The lower and upper limits for the solution space of the problem are defined by the variables $L_{b}$ and $U_{b}$ in Equation 7. The rand in Equation 7 and Equation 8 represents the randomness. In this mechanism, the position of the first individual is updated with Equation 8a, while the positions of the other individuals are updated using Equation 8b [40].

\subsection{Somersault Foraging}

Another foraging strategy used by manta rays during foraging is the somersault strategy [40]. In this strategy, manta ray individuals try to pull the plankton towards the manta rays by making a series of somersaults as they circle around the position of the high-dense planktons. This mechanism is mathematically implemented as stated in Equation 9 for all individuals of the population [40].

$$
x_{i}^{d}(t+1)=x_{i}^{d}(t)+S \cdot\left(r_{2} \cdot x_{\text {best }}^{d}-r_{3} \cdot x_{i}^{d}(t)\right), i=1, \ldots, N
$$

The variables $S, r_{2}$, and $r_{3}$ in Equation 9 represent the somersault coefficient and two randomly chosen numbers between 0 and 1 , respectively. Somersault coefficient $S$ is a coefficient that defines the somersault range of manta rays and can be selected as 2 [40].

\subsection{Application of the Lévy-Flight Technique to MRFO}

The Lévy-flights method, proposed by the French mathematician Paul Lévy, is inspired by the natural ability that creatures in nature follow during hunting and foraging [36, 41]. When the solution quality in the current position cannot be increased during foraging, the new position is searched by moving quickly to a distant location, and this process is repeated until the best position is found [36, 41]. The Lévy-flights mechanism is used during the somersault foraging process as it offers easy applicability to the entire population in improving the best position of manta rays in the MRFO algorithm [40]. In this study, a randomly selected number was used as the decision value in implementing the Lévy-flights mechanism. The classical somersault strategy was used if the decision value had been lower than 0.5; otherwise, the Lévy-flight mechanism was used. By applying the Lévy-flight mechanism, the new positions of the individuals were calculated using Equation 10.

$$
x_{i}^{d}(t+1)=\operatorname{Lévy}^{d} \cdot\left(x_{i}^{d}(t)-x_{\text {best }}^{d}(t)\right), i=1, \ldots, N
$$

The Lévy ${ }^{d}$ parameter given in Equation 10 represents the step size of the Lévy-flight search mechanism and is formulated in Equation 11 [36, 41].

$$
\text { Lévyd }=c_{l e ́ v y} \frac{r_{4} r_{5}}{\left|r_{6}\right|^{\frac{1}{\lambda}}}
$$

$C_{\text {lévy }}$ and $\lambda$ parameters given in Equation 11 are constants, and the values of the constants were chosen as 0.001 and 1, respectively. In Equation 11, $r_{4}, r_{5}$, and $r_{6}$ are parameters that provide randomness. The $r_{4}$ parameter is a random number in the range of $\left[L_{b}, U_{b}\right]$, the $r_{5}$ parameter is a random number chosen according to the Gaussian distribution in the range of $\left[0, \sigma^{2}\right]$, and the $r_{6}$ parameter is a random number chosen according to the Gaussian distribution in the range of $[0,1]$ [36, 41]. As the Gamma function is a function defined as $\Gamma(x)=(x-1)$ !, variance $\left(\sigma^{2}\right)$ was calculated by the expression given in Equation $12[36,41]$. 


$$
\sigma^{2}=\left(\frac{\Gamma(1+\lambda) \cdot \sin \left(\frac{\pi \lambda}{2}\right)}{\Gamma\left(\frac{1+\lambda}{2}\right) \cdot \lambda \cdot 2^{\frac{\lambda-1}{2}}}\right)^{\frac{1}{\lambda}}
$$

Equation 12 is based only on the constant $\lambda$, and the value of $\lambda$ was chosen as 1 . The pseudocode for the MRFO algorithm [40, 42], including the Lévy-flight mechanism, is shown in Algorithm 1.

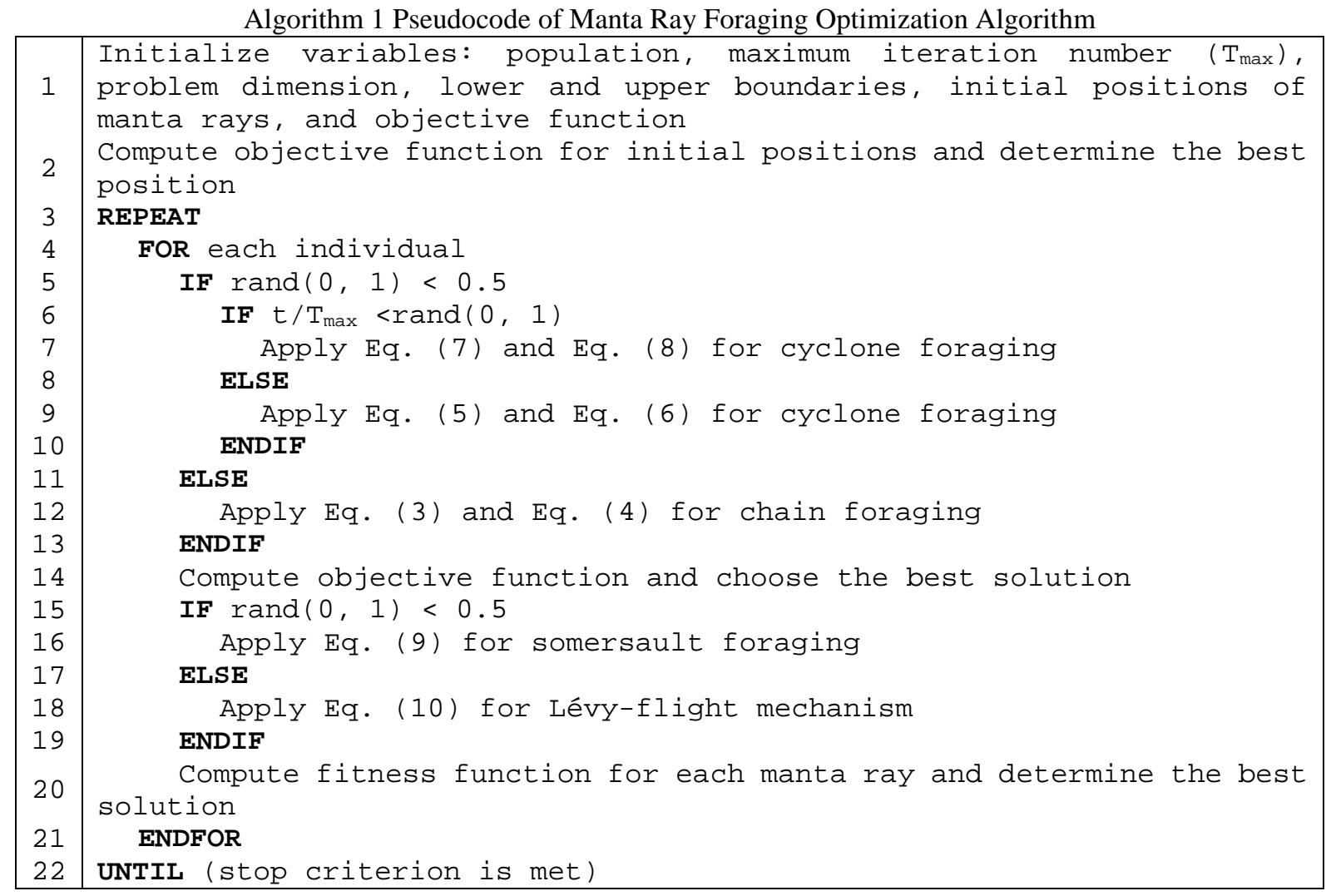

Algorithm 1 Pseudocode of Manta Ray Foraging Optimization Algorithm

\section{Optimizing the DNN-based Model using the Improved MRFO Algorithm}

The simulation study for 324 different CCMA designs with various physical and electrical values given in Table 1 was carried out utilizing a FIT-based full-wave electromagnetic solver, and the gain and $S_{11}$ values were obtained for the desired frequency range. The $S_{11}$ value is defined as the reflection coefficient at a specific frequency, while the gain is an important parameter that includes all the losses of the antenna. In order to obtain the gain and $S_{11}$ values, a certain number of frequencies $\left(n_{f}\right)$ must be determined between the lower frequency $\left(f_{l}\right)$ and the upper frequency $\left(f_{u}\right)$ in a particular frequency range. Furthermore, $f_{l}, f_{u}$, and $n_{f}$ were chosen as $1 \mathrm{GHz}, 5 \mathrm{GHz}$, and 70, respectively, to limit the size of the data set and obtain a sufficient solution space for the model. Studies on CCMAs generally focus on the frequency band between $1 \mathrm{GHz}$ and $10 \mathrm{GHz}$ in the literature [7, 29, 33, 38], which has been influential in choosing the frequency range. A seven-layered DNN-based neural model was subsequently constructed, consisting of seven inputs and two outputs, as shown in Figure 3. 


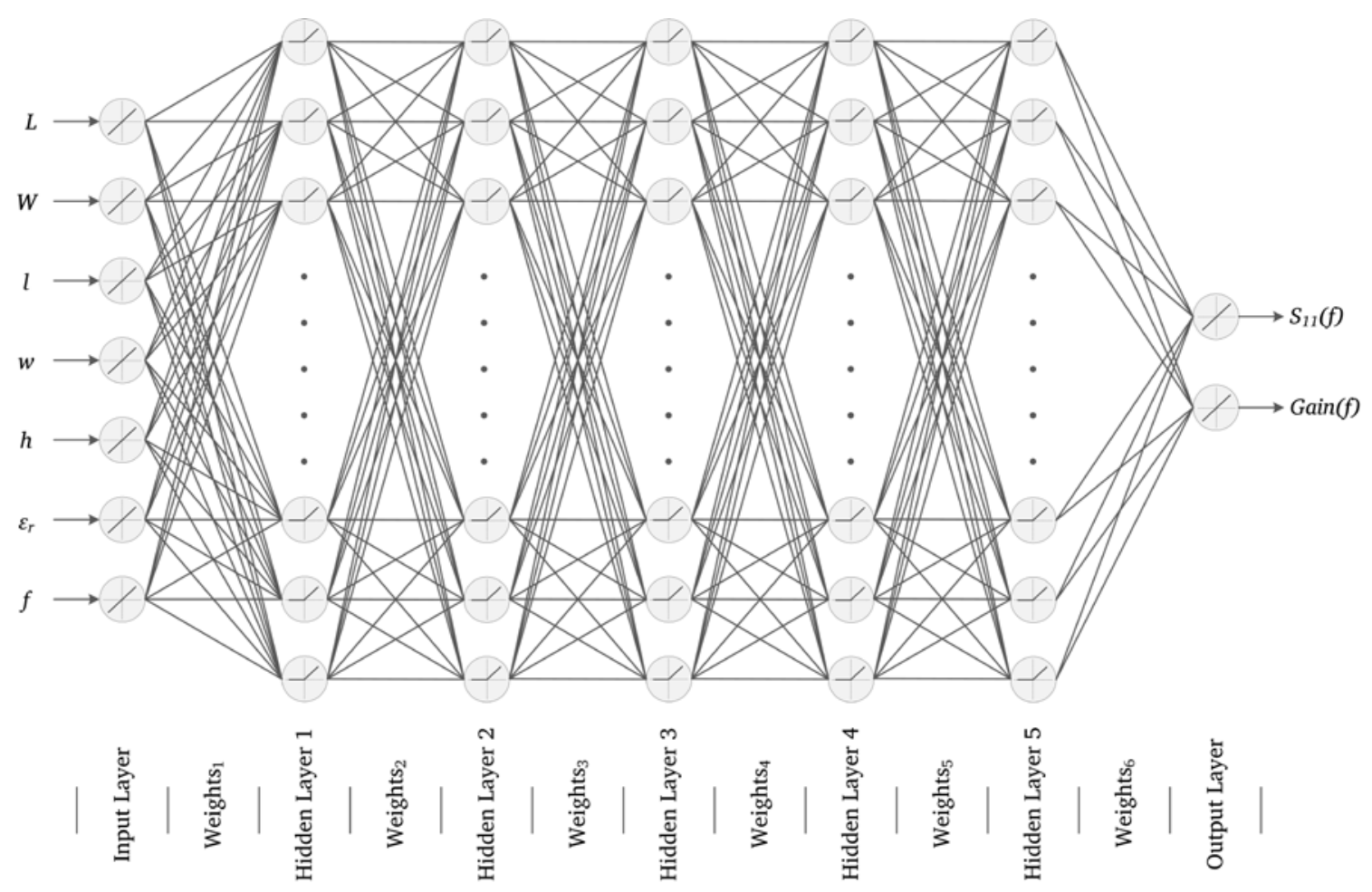

Figure 1 The proposed DNN-based model to predict the gain and $\mathrm{S}_{11}$ for a CCMA.

As seen in Figure 3, the proposed model consists of an input layer with seven neurons, five hidden layers with 36 neurons for each one, and an output layer with two neurons to estimate the gain and $S_{11}$. For each neuron in the hidden layers, the rectified linear unit activation function was used. In the training phase, data from 259 of the designed CCMAs was used, while the remaining data was used to test the model. Thus, 18144 pieces of $S_{11}$ data were used for training the proposed model, while 4536 pieces of data were used to test the model. The training of the model can be defined as the determination of the weights and biases between layers of the DNN model that can estimate output values with the least error by using the input data set. In this study, the training of the proposed neural model was carried out using the improved MRFO algorithm [40] with the Lévy-flight mechanism [36, 41]. The weight matrices and bias vectors of the proposed neural structure constitute the problem variables of the optimization algorithm. In order to be used in the optimization process, the weight matrices and bias vectors were first transformed into a one-dimensional vector, and a single problem vector was created by combining these vectors. While the length of the problem vector determines the problem dimension, each vector element represents the problem variable to be optimized. In each iteration, the best solution computed by the algorithm was replaced by the weight matrices and bias vectors in the model, and predictions were made for the data set. Afterward, the MSE was calculated between the output values and the estimated values. The optimization algorithm continued the iterative process by searching for better solutions for the problem variables based on the mean squared error. In this study, the weight and bias matrices of the proposed model were optimized on an Intel I7-9750H CPU with 32 GB RAM. The flowchart of the optimization process is shown in Figure 4.

\section{Numerical Results and Discussion}

In this study, a seven-layered neural model with 5690 trainable parameters was proposed, and the model was trained during 5000 epochs with 18144 data. The training and test performances of the model were measured with the $\mathrm{R}^{2}$ score, MAE, and MSE given in Equation 13, Equation 14, and Equation 15, respectively. 
Mustafa Berkan BICER

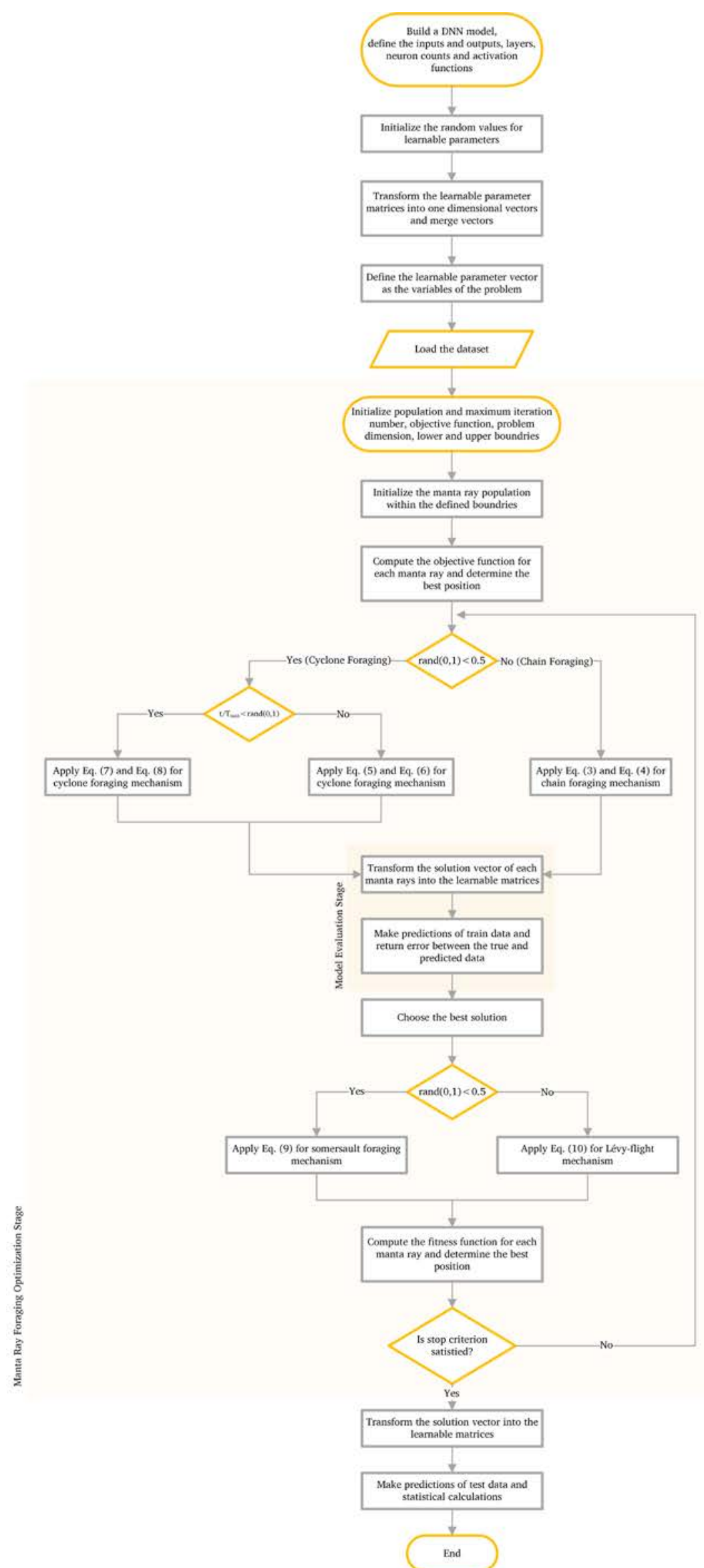

Figure 4 The flowchart of the MRFO-based weight and bias optimization 


$$
\begin{gathered}
R^{2}=1-\frac{\sum_{i=1}^{n}\left(y_{t}-y_{p}\right)^{2}}{\sum_{i=1}^{n}\left(y_{t}-y_{t, \text { mean }}\right)^{2}} \\
M A E=\frac{\sum_{i=1}^{n}\left|y_{t}-y_{p}\right|}{n} \\
M S E=\frac{\sum_{i=1}^{n}\left(y_{t}-y_{p}\right)^{2}}{n}
\end{gathered}
$$

In Equation 13 through 15, the terms $n, y_{t}, y_{p}$, and $y_{t, \text { mean }}$ represent the number of data, the target value, the predicted value, and the mean of the predicted value, respectively. The best performance metrics for training and test processes were achieved when the seed value of the random number generator was chosen as 8025443 . Table 2 presents the performance metrics of the proposed model.

Table 1 Performance metrics of the proposed model for training, test, and the entire data

\begin{tabular}{llll}
\hline Metrics & Train & Test & Entire \\
\hline Length of Data Set & 18144 & 4536 & 22680 \\
\hline $\mathrm{R}^{2}$ score & 0.925 & 0.922 & 0.950 \\
\hline MAE & 0.620 & 0.581 & 0.612 \\
\hline MSE & 3.862 & 2.446 & 3.579 \\
\hline
\end{tabular}

The performance metrics given in Table 2 were calculated for 70 frequency points using the physical and electrical properties of each antenna. From the table, it can be seen that the performance metrics of the proposed model for the test data are slightly better than the training data. For the current data set, the $\mathrm{R}^{2}$ score was found to be quite close to 1 , while MAE and MSE metrics were quite acceptable. According to the results, it is noted that the proposed model is not over-fitted. Therefore it can be expected to predict near-accurate results for data not included in the solution space. The comparison of the desired and predicted $S_{11}$ curves of randomly chosen four different CCMAs operating between 1 $\mathrm{GHz}$ and $5 \mathrm{GHz}$ is illustrated in Figure 5.

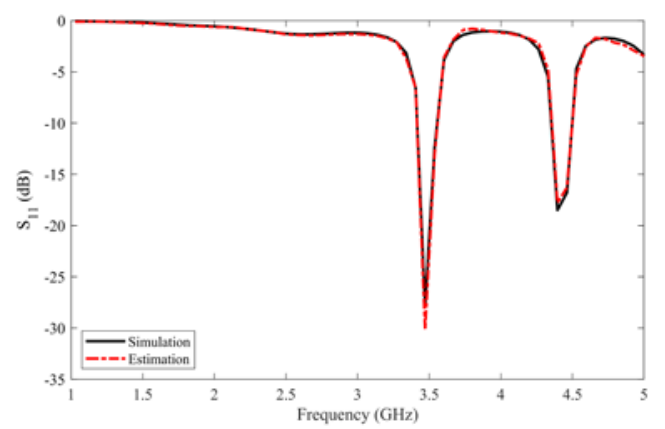

(a)

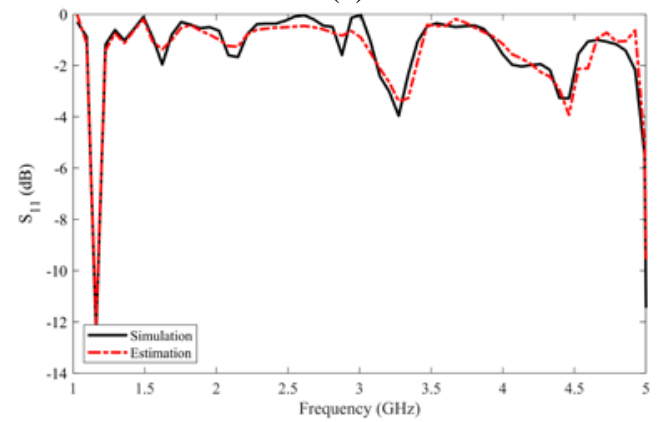

(c)

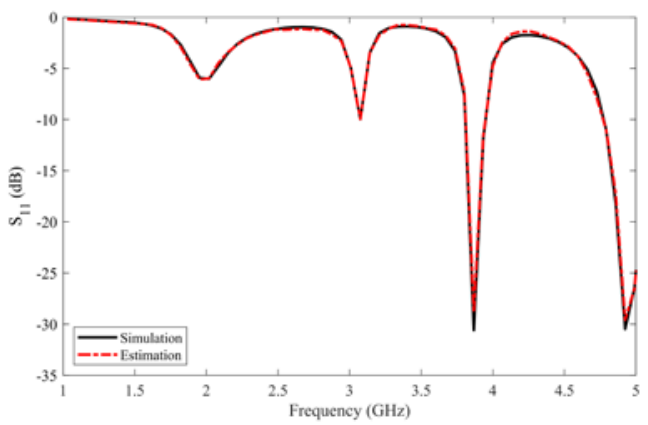

(b)

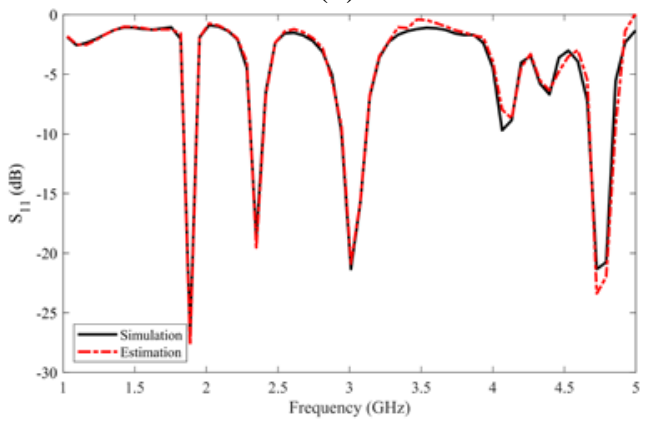

(d)

Figure 5 The simulated and estimated $\mathrm{S}_{11}$ curves for the randomly chosen four CCMAs, Antenna (a) \#1, (b) \#2, (c) \#3, (d) \#4 (detailed in Table 3) 
Table 2 Values of the physical and electrical parameters for the randomly chosen antennas for $\mathrm{S}_{11}$ curves

\begin{tabular}{lcccc}
\hline & \multicolumn{4}{c}{ Values for antennas } \\
\hline Properties & $\mathbf{\# 1}$ & $\mathbf{\# 2}$ & $\mathbf{\# 3}$ & $\mathbf{\# 4}$ \\
\hline $\mathrm{L}(\mathrm{mm})$ & 30 & 45 & 60 & 75 \\
\hline $\mathrm{W}(\mathrm{mm})$ & 20 & 30 & 40 & 50 \\
\hline $\mathrm{l}(\mathrm{mm})$ & 3 & 17 & 40 & 28 \\
\hline $\mathrm{w}(\mathrm{mm})$ & 9 & 13 & 30 & 21 \\
\hline $\mathrm{h}(\mathrm{mm})$ & 3 & 4.5 & 1.6 & 4.5 \\
\hline$\varepsilon_{\mathrm{r}}$ & 4.28 & 2.33 & 6.15 & 2.33 \\
\hline $\mathrm{R}^{2}$ & 0.993 & 0.997 & 0.954 & 0.982 \\
\hline
\end{tabular}

For randomly chosen four CCMAs having different outer dimensions $L \times \mathrm{x}$, the predicted $S_{11}$ values between $1 \mathrm{GHz}$ and $5 \mathrm{GHz}$ frequencies were obtained using the model, and the target and estimated $S_{11}$ values were compared in Figure 5 (a) - (d). The $S_{11}$ curves illustrated in Figure 5 (a) and (b) demonstrate great harmony between the estimated $S_{11}$ values and the target ones. As can be seen from Figure 5 (b) and (d), the increase in the values of the physical parameters causes acceptable discrepancies between the estimated and simulated values. In Figure 5 (c), the estimated values are consistent with the simulated values, but it is seen that the errors have increased slightly. The comparative curves for simulated and estimated gain values for four randomly chosen CCMAs are given in Figure 6.

Table 3 Values of the physical and electrical parameters for the randomly chosen antennas for gain curves

\begin{tabular}{lcccc}
\hline & \multicolumn{4}{c}{ Values for antennas } \\
\hline Properties & $\mathbf{\# 1}$ & $\mathbf{\# 2}$ & $\mathbf{\# 3}$ & $\mathbf{\# 4}$ \\
\hline $\mathrm{L}(\mathrm{mm})$ & 30 & 45 & 60 & 75 \\
\hline $\mathrm{W}(\mathrm{mm})$ & 20 & 30 & 40 & 50 \\
\hline $\mathrm{l}(\mathrm{mm})$ & 3 & 17 & 40 & 28 \\
\hline $\mathrm{W}(\mathrm{mm})$ & 9 & 13 & 30 & 21 \\
\hline $\mathrm{h}(\mathrm{mm})$ & 3 & 4.5 & 1.6 & 4.5 \\
\hline$\varepsilon \mathrm{r}$ & 4.28 & 2.33 & 6.15 & 2.33 \\
\hline $\mathrm{R}^{2}$ & 0.982 & 0.968 & 0.995 & 0.996 \\
\hline
\end{tabular}

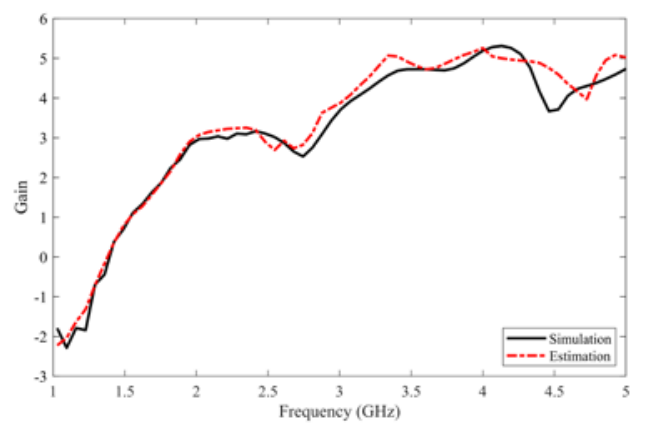

(a)

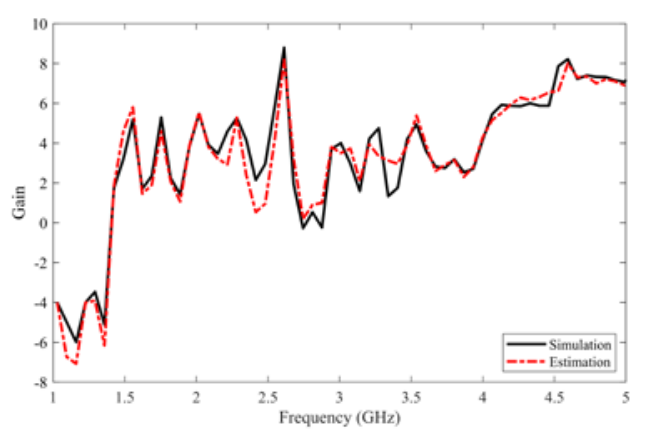

(c)

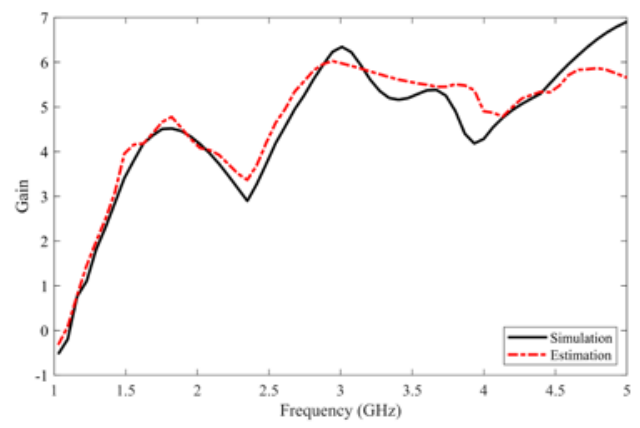

(b)

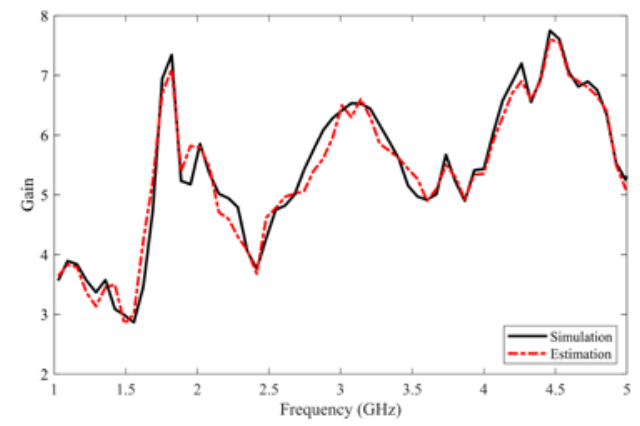

(d)

Figure 6 The simulated and estimated gain curves for the randomly chosen four CCMAs, Antenna (a) \#1, (b) \#2, (c) \#3, (d) \#4 (detailed in Table 4) 
As shown in Figure 6, the model made relatively more errors for the estimation of the gain values than the $S_{11}$ values. Due to the use of a single model with two outputs and the limited number of data, acceptable mismatches occurred between the estimated data and the simulation data. As seen in Figure 5 and Figure 6, while the harmony is high at low frequencies, the discrepancy between the estimated data and the simulation data increases as the frequency increases. As mentioned in the literature review, most of the studies related to CCMAs have focused on optimizing the antenna designs for specific applications and obtaining mathematical models that usually provide the resonant frequency $[3,5,6$, 17-34]. Although this study is not directly related to the estimation of resonant frequencies of CCMAs, the resonant frequencies in dominant mode $\left(\mathrm{TM}_{010}\right)$ were indirectly estimated by the interpretation of the $S_{11}$ curves, and the comparison between the results obtained in this study and the results in the literature $[7,23]$ is shown in Table 5 . Comparisons of resonant frequencies are made based on the simulation results presented by [5].

Table 5 Comparative results and percentage errors on resonant frequencies

\begin{tabular}{|c|c|c|c|c|c|c|c|c|c|c|c|c|c|c|c|}
\hline \multirow{4}{*}{ \# } & \multirow{2}{*}{\multicolumn{2}{|c|}{$\begin{array}{c}\text { Slot } \\
\text { dimensions } \\
(\mathrm{mm})\end{array}$}} & \multicolumn{7}{|c|}{ Resonant frequencies (GHz) } & \multirow{2}{*}{\multicolumn{6}{|c|}{ Percentage errors (\%) }} \\
\hline & & & \multirow{3}{*}{$\begin{array}{c}\text { Simulated } \\
{[5]}\end{array}$} & \multirow{3}{*}{$\begin{array}{c}\text { Estimated } \\
\text { This } \\
\text { Study }\end{array}$} & \multirow{3}{*}[22]{} & \multicolumn{4}{|c|}{ Calculated } & & & & & & \\
\hline & & & & & & & & [5] & & \multirow{2}{*}{$\begin{array}{l}\text { This } \\
\text { Study }\end{array}$} & \multirow{2}{*}{ [22] } & \multirow{2}{*}{ [6] } & \multicolumn{3}{|c|}{ [5] } \\
\hline & 1 & $\mathbf{w}$ & & & & [6] & $\# 1$ & $\# 2$ & \#3 & & & & $\# 1$ & $\# 2$ & \#3 \\
\hline 1 & 5 & 5 & 1.562 & 1.559 & 1.562 & 1.657 & 1.502 & 1.630 & - & 0.192 & 0.000 & 6.082 & 3.841 & 4.353 & - \\
\hline 2 & 10 & 10 & 1.445 & 1.452 & 1.426 & 1.497 & 1.398 & 1.408 & - & 0.484 & 1.315 & 3.599 & 3.253 & 2.561 & - \\
\hline 3 & 15 & 15 & 1.286 & 1.271 & 1.280 & 1.334 & 1.309 & 1.241 & - & 1.166 & 0.467 & 3.732 & 1.788 & 3.499 & - \\
\hline 4 & 20 & 20 & 1.130 & 1.102 & 1.136 & 1.178 & 1.231 & 1.111 & 1.002 & 2.478 & 0.531 & 4.248 & 8.938 & 1.681 & 11.327 \\
\hline 5 & 25 & 25 & 0.991 & 0.998 & 1.000 & 1.035 & 1.164 & 1.008 & 0.928 & 0.706 & 0.908 & 4.440 & 17.457 & 1.715 & 6.357 \\
\hline 6 & 40 & 30 & 0.899 & 0.892 & 0.890 & 0.924 & - & 0.893 & 0.856 & 0.779 & 1.001 & 2.781 & - & 0.667 & 4.783 \\
\hline 7 & 5 & 30 & 0.929 & 0.916 & 0.931 & 0.963 & - & 1.029 & 0.904 & 1.399 & 0.215 & 3.660 & - & 10.764 & 2.691 \\
\hline 8 & 10 & 30 & 0.887 & 0.881 & 0.911 & 0.938 & - & - & 0.896 & 0.676 & 2.706 & 5.750 & - & - & 1.015 \\
\hline \multirow[t]{2}{*}{9} & 2 & 30 & 0.964 & 0.959 & 0.946 & 0.982 & - & - & 0.910 & 0.519 & 1.867 & 1.867 & - & - & 5.602 \\
\hline & & & & & \multicolumn{5}{|c|}{ Average percentage errors (APE) } & 0.933 & 1.001 & 4.018 & 7.055 & 3.606 & 5.296 \\
\hline
\end{tabular}

$\mathrm{L}=60 \mathrm{~mm}, \mathrm{~W}=40 \mathrm{~mm}, \mathrm{~h}=1.59 \mathrm{~mm}, \varepsilon_{\mathrm{r}}=2.33$

- Not available.

The physical dimensions of antennas between \#1 - \#8 in Table 5 are within the range of dimensions, while \#9 is outside the range. The dominant resonant frequencies estimated using the proposed model, however, were entirely in line with the literature, and the percentage error (PE) values were calculated to be considerably small. For literature data, the average PE (APE) value was calculated as $0.933 \%$ for the estimated resonant frequencies. Afterward, a CCMA with outer dimensions $30 \times 20 \mathrm{~mm}^{2}$ and slot dimensions $3 \times 7 \mathrm{~mm}^{2}$ was fabricated using Rogers RT/duroid 5870 substrate with a thickness of 1.57 $\mathrm{mm}$ [23]. The antenna was fed from the bottom-left concerning the $L$-axis using a 90-degree SMA connector. The $S_{11}$ of the CCMA was measured by using Agilent E5071B ENA RF Series Network Analyzer. The estimated $S_{11}$ curve using the proposed model was compared with the simulated and measured values of the fabricated antenna, and the comparative curves are shown in Figure 7.

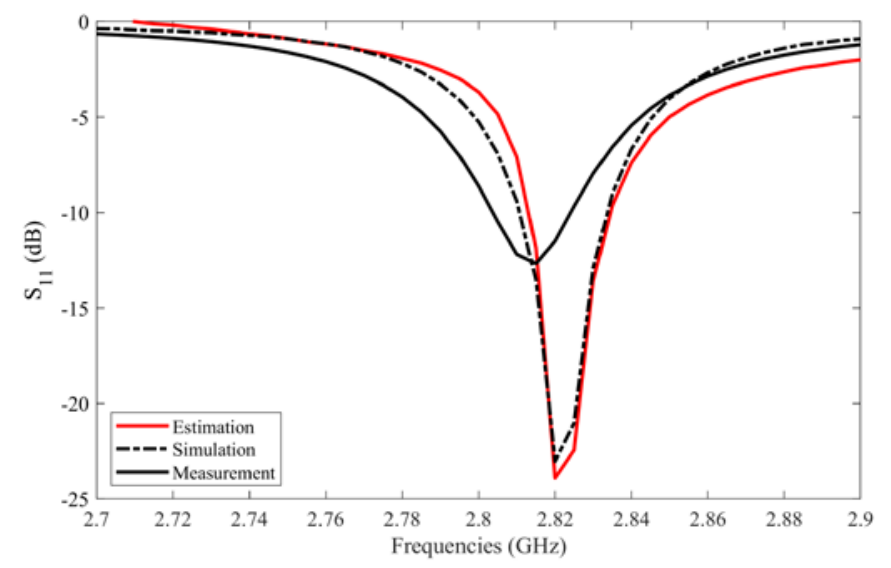

Figure 7 Comparison of the $\mathrm{S}_{11}$ curves for the CCMA $\left(\varepsilon_{r}=2.33\right)$ 
While the $S_{11}$ curve obtained in the simulation and the estimated $S_{11}$ curve obtained by the model are in harmony, the measured $S_{11}$ curve of the fabricated CCMA is similar in shape but shifts slightly to the left of the frequency axis, as shown in Figure 7. Since the model is trained using the data set obtained from the simulation, it yielded a similar result to the data obtained by the simulation, though with slight inaccuracy. The training data set needs to be expanded, and the number of layers in the model needs to be increased in order to achieve better results. However, since the study aimed to ensure that the training data set is precisely learned by the model, the result shown in Figure 7 is given only for the purpose of benchmarking.

\section{Conclusion}

Recent studies in the literature have focused on estimating the characteristics of microstrip antennas, such as resonant frequencies and gains, using machine learning methods. This study was focused on the estimation of $S_{11}$ and gain values in a certain frequency range instead of estimating the resonant frequencies of microstrip antennas. For this purpose, a DNN-based neural model was proposed and optimized using the MRFO algorithm. The gain and $S_{11}$ values of CCMAs, operating within the frequency range from $1 \mathrm{GHz}$ to $5 \mathrm{GHz}$, were obtained for specific physical and electrical properties to build the data set. The weight matrices and bias vectors of the proposed model were optimized using this data set. $\mathrm{R}^{2}$ score that shows the success of the model for training and test data was obtained as 0.925 and 0.922 , respectively. Besides, the average percentage error (APE) for the prediction of resonant frequency between the obtained data with the literature data was calculated as $0.933 \%$. It is seen that extending the data set by making more antenna designs having various physical and electrical characteristics and by making more simulations for smaller frequency steps in the wider frequency band will increase the performance of the model. The results show that DNN-based models can be utilized successfully in estimating the gain and $S_{11}$ values of CCMAs. The proposed model gives fast and practical results, and it can be used as a computer-aided design (CAD) tool by antenna designers and researchers.

\section{Authors' Contributions}

All authors contributed equally to the study.

\section{Statement of Conflicts of Interest}

There is no conflict of interest between the authors.

\section{Statement of Research and Publication Ethics}

The author declares that this study complies with Research and Publication Ethics.

\section{References}

[1] J. Q. Howell, “Microstrip Antennas,” IEEE Transactions on Antennas and Propagation, vol. 23, pp. 90-93, 1975.

[2] C. A. Balanis, Antenna Theory: Analysis and Design. Wiley \& Sons, Inc., 2016.

[3] R. N. Tiwari, P. Singh, and B. K. Kanaujia, "A modified microstrip line fed compact UWB antenna for WiMAX/ISM/WLAN and wireless communications," AEU - International Journal of Electronics and Communications, vol. 104, pp. 58-65, 2019.

[4] G. Kumar, and K. P. Ray, Broadband Microstrip Antennas. Artech House, 2003.

[5] A. A. Deshmukh, and G. Kumar, "Formulation of resonant frequency for compact rectangular microstrip antennas," Microwave and Optical Technology Letters, vol. 49, no. 2, pp. 498-501, 2007.

[6] A. H. Yuzer, and C. Seker, "4G C-shaped compact microstrip antenna design and production," Pamukkale University Journal of Engineering Sciences, vol. 23, no. 5, pp. 532-535, 2017. 
[7] A. Akdagli, M. B. Bicer, and S. Ermis, "A novel expression for resonant length obtained by using artificial bee colony algorithm in calculating resonant frequency of C-shaped compact microstrip antennas,” Turkish Journal of Electrical Engineering and Computer Sciences, vol. 19, no. 4, pp. 597-606, 2011.

[8] A. K. Bhattacharyya, and R. Garg, "Generalized transmission line model for microstrip patches,” Microwaves Optics and Antennas, vol. 132, no. 2, pp. 93-98, 1985.

[9] W. Richards, Y. Lo, and D. Harrison, "An improved theory for microstrip antennas and applications," IEEE Transactions on Antennas and Propagation, vol. 29, no. 1, pp. 38-46, 1981.

[10] A. Taflove, and S. C. Hagness, Computational Electrodynamics: The Finite-Difference TimeDomain Method. Artech House, 2005.

[11] S. C. Gao, L. W. Li, M. S. Leong, T. S. Yeo, “Analysis of an H-shaped patch antenna by using the FDTD method,” Progress In Electromagnetics Research, vol. 34, pp. 165-187, 2001.

[12] J. L. Volakis, A. Chatterjee, and L. C. Kempel, Finite Element Method Electromagnetics: Antennas, Microwave Circuits, and Scattering Applications. Wiley, 1998.

[13] T. Weiland, and M. Clemens, "Discrete electromagnetism with the finite integration technique," Progress In Electromagnetics Research, vol. 32, pp. 65-87, 2001.

[14] T. Weiland, "A discretization method for the solution of Maxwell's equations for six-component fields," AEU - International Journal of Electronics and Communications, vol. 31, no. 3, pp. 116120, 1977.

[15] R. F. Harrington. Field Computation by Moment Methods. Wiley, 1993.

[16] E. H. Newman, and P. Tulyathan, “Analysis of microstrip antennas using moment methods," IEEE Transactions on Antennas and Propagation, vol. 29, no. 1, pp. 47 - 53, 1981.

[17] X. Cao, Y. Xia, L. Wu, and L. Lang, “A compact quad-band ring string-shaped antenna,” AEU International Journal of Electronics and Communications, vol. 111, pp. 152910, 2019.

[18] A. T. Mobashsher, K. S. Bialkowski, and A. M. Abbosh, "Design of compact cross-fed threedimensional slot-loaded antenna and its application in wideband head imaging system," IEEE Antennas and Wireless Propagation Letters, vol. 15, pp. 1856 - 1860, 2016.

[19] D. Ustun, and A. Akdagli, "Design of a dual-wideband monopole antenna by artificial bee colony algorithm for UMTS, WLAN, and WiMAX applications," International Journal of Microwave and Wireless Technologies, vol. 9, no. 5, pp. 1197-1208, 2017.

[20] J. M. Felicio, J. M. Bioucas-Dias, J. R. Costa, and C. A. Fernandes, "Antenna design and nearfield characterization for medical microwave imaging applications," IEEE Transactions on Antennas and Propagation, vol. 67, no. 7, pp. 4811 - 4824, 2019.

[21] A. Akdagli, C. Ozdemir, S. Yamacli, and C. C. Arcasoy. "Improved formulas for the resonant frequencies of dual frequency arrow shaped compact microstrip antenna," Microwave and Optical Technology Letters, vol. 50, no. 1, pp. 62-65, 2008.

[22] V. S. Chintakindi, S. S. Pattnaik, O. P. Bajpai, S. Devi, P. K. Patra, and K. M. Bakwad, "Resonant frequency of equilateral triangular microstrip patch antenna using particle swarm optimization technique," 2008 International Conference on Recent Advances in Microwave Theory and Applications, 2008.

[23] A. Toktas, M. B. Bicer, A. Akdagli, and A. Kayabasi, "Simple formulas for calculating resonant frequencies of $\mathrm{C}$ and $\mathrm{H}$ shaped compact microstrip antennas obtained by using artificial bee colony algorithm," Journal of Electromagnetic Waves and Applications, vol. 25, no. 11-12, pp. 17181729, 2011.

[24] M. Biswas, and M. Dam, "Closed-form model to determine the co-axial probe reactance of an equilateral triangular patch antenna," International Journal of Microwave and Wireless Technologies, vol. 10, no. 7, pp. 801 - 813, 2018.

[25] B. Sami, B. Siham, and F. Tarek, "Analysis of a circular microstrip antenna on isotropic or uniaxially anisotropic substrate using neurospectral approach,” COMPEL: The International Journal for Computation and Mathematics in Electrical and Electronic Engineering, vol. 33, no. 1/2, pp. 567-580, 2014.

[26] L. Barkat, S. Bedra, T. Fortaki, and R. Bedra, "Neurospectral computation for the resonant characteristics of microstrip patch antenna printed on uniaxially anisotropic substrates," International Journal of Microwave and Wireless Technologies, vol. 9, no. 3, pp. 613-620, 2017. 
[27] A. Kayabasi, A. Toktas, A. Akdagli, M. B. Bicer, and D. Ustun, "Applications of ANN and ANFIS to predict the resonant frequency of L-shaped compact microstrip antennas," Applied Computational Electromagnetics Society Journal, vol. 29, no. 6, 2014.

[28] A. Kayabasi, M. B. Bicer, A. Akdagli, and A. Toktas, "Computing resonant frequency of H-shaped compact microstrip antennas operating at UHF band by using artificial neural networks," Journal of the Faculty of Engineering and Architecture of Gazi University, vol. 26, no. 4, 2011.

[29] D. Ustun, A. Toktas, and A. Akdagli, "Deep neural network-based soft computing the resonant frequency of E-shaped patch antennas," AEU - International Journal of Electronics and Communications, vol. 102, pp. s54-61, 2019.

[30] M. Kaur, and J. S. Sivia, "Giuseppe Peano and Cantor set fractals based miniaturized hybrid fractal antenna for biomedical applications using artificial neural network and firefly algorithm," International Journal of RF and Microwave Computer-Aided Engineering, vol. 30, no. 1, 2020.

[31] K. Y. Kapusuz, H. Tora, and S. Can, "Neural network based estimation of resonant frequency of an equilateral triangular microstrip patch antenna," Tehnicki Vjesnik, vol. 20, no. 6, pp. 955-960, 2013.

[32] S. Can, K. Y. Kapusuz, and E. Aydin, "Neural network based resonant frequency solver for rectangular-shaped shorting pin-loaded antennas," Microwave and Optical Technology Letters, vol. 55, no. 12, pp. 3025-3028, 2013.

[33] J. Singh, G. Singh, S. Kaur, and B. S. Sohi, "Performance analysis of different neural network models for parameters estimation of coaxial fed $2.4 \mathrm{GHz}$ E-shaped microstrip patch antenna," 2015 2nd International Conference on Recent Advances in Engineering and Computational Sciences, 2015.

[34] T. M. Neebha, and M. Nesasudha, "Artificial neural network based design of a microstrip patch antenna for RADAR applications," Proceedings of the International Conference on Recent Advances in Aerospace Engineering, 2017.

[35] D. Karaboga, and B. Basturk, "A powerful and efficient algorithm for numerical function optimization: artificial bee colony (ABC) algorithm,” Journal of Global Optimization, vol. 39, no. 3, pp. 459-471, 2007.

[36] X. S. Yang, Engineering Optimization: An Introduction with Metaheuristic Applications. Wiley, 2010.

[37] A. Kaveh, and T. Bakhshpoori, Metaheuristics: Outlines, MATLAB Codes and Examples. Springer, 2019.

[38] P. K. Abbassi, N. M. Badra, A. M. M. A. Allam, and A. El-Rafei, "WiFi antenna design and modeling using artificial neural networks," Proceedings of 2019 International Conference on Innovative Trends in Computer Engineering, 2019.

[39] X. S. Yang, "Firefly algorithms for multimodal optimization," Lecture Notes in Computer Science, 2009.

[40] W. Zhao, Z. Zhang, L. Wang, "Manta ray foraging optimization: An effective bio-inspired optimizer for engineering applications," Engineering Applications of Artificial Intelligence, vol. 87, pp. 103300, 2020.

[41] Y. Li, X. Li, J. Liu, and X. Ruan, "An improved bat algorithm based on lévy flights and adjustment factors," Symmetry, vol. 11, no. 7, pp. 925, 2019.

[42] O. E. Turgut, "A novel chaotic manta-ray foraging optimization algorithm for thermo-economic design optimization of an air-fin cooler,” SN Applied Sciences, vol. 3, no. 1, pp. 3, 2021. 